\title{
Diacronie
}

Studi di Storia Contemporanea

$N^{\circ} 25,1 \mid 2016$

"Se creare è definire"

\section{Nikolai Wehrs, Protest der Professoren. Der «Bund Freiheit der Wissenschaft» in den 1970er Jahren}

\section{Alessandro Stoppoloni}

\section{(2) OpenEdition}

\section{Edizione digitale}

URL: http://journals.openedition.org/diacronie/3896

DOI: 10.4000/diacronie.3896

ISSN: 2038-0925

\section{Editore}

Association culturelle Diacronie

\section{Notizia bibliografica digitale}

Alessandro Stoppoloni, « Nikolai Wehrs, Protest der Professoren. Der «Bund Freiheit der Wissenschaft» in den 1970er Jahren », Diacronie [Online], № 25, 1 | 2016, documento 14, Messo online il 29 mars 2016, consultato il 24 septembre 2020. URL : http://journals.openedition.org/diacronie/3896 ; DOI : https:// doi.org/10.4000/diacronie.3896 


\title{
RECENSIONE:
}

\section{Nikolai WEHRS, Protest der Professoren. Der «Bund Freiheit der Wissenschaft» in den 197oer Jahren, Göttingen, Wallstein Verlag,} 2014, 539 pp.

\author{
a cura di Alessandro STOPPOLONI *
}

Nell'immaginario collettivo l'anno 1968 e quelli che l'hanno immediatamente preceduto e seguito sono legati ai giovani e, soprattutto in alcuni casi, agli studenti. Se molti sono stati gli studi che, sia a livello internazionale sia a livello nazionale, si sono occupati di analizzare il ruolo di questi ultimi nei movimenti di protesta, molta meno attenzione è stata finora riservata a coloro che con gli studenti avevano quotidianamente a che fare per ragioni professionali, cioè i professori ${ }^{1}$. Il lavoro di Nikolai Wehrs, oggi collaboratore presso la cattedra di storia contemporanea dell'università di Costanza (Germania), cerca di colmare in parte questa lacuna, almeno in relazione alla storia tedesca. Il libro è frutto della ricerca di dottorato di Wehrs e si occupa della storia del Bund Freiheit der Wissenschaft (BFW), un'organizzazione fondata nel 1970 su iniziativa di alcuni professori che si proponeva di contrastare alcune tendenze nate a partire dal movimento studentesco viste come un pericolo per la

${ }^{1}$ Gli studi disponibili sui professori universitari e il ' 68 sono spesso stati realizzati su base locale. In questo senso si può consultare, limitatamente alla storia dell'università di Palermo dalla caduta del fascismo fino alla fine del XX secolo, VIOLA, Paolo, Oligarchie, Roma, Donzelli, 2006. Sull'università di Roma si rimanda a SOCRATE, Francesca (a cura di), Un altro Sessantotto. La protesta nella memoria dei docenti dell'Università di Roma "La Sapienza", 2 voll., Roma, Biblink, 2008. Su questioni di metodologia legate all'uso di fonti orali per lavorare su un simile tema (anche in questo caso legate a un caso specifico) si può consultare AGOSTINI, Giovanni, GIORGI, Andrea, MINEO, Leonardo (a cura di), La memoria dell'Università. Le fonti orali per la storia dell'Università degli studi di Trento (1962-1972), Bologna, Il Mulino, 2014. Altri spunti possono derivare dagli atti del convegno Le istituzioni universitarie e il Sessantotto tenutosi a Pisa nel 2011 e, su questioni più legate allo studio delle fonti, a Bologna nel 2012: BRECCIA, Alessandro (a cura di), Le istituzioni universitarie e il Sessantotto, Bologna, Clueb, 2013. Per le segnalazioni si ringraziano la professoressa Marica Tolomelli dell'Università di Bologna e la professoressa Francesca Socrate dell'Università Sapienza di Roma. 
libertà di insegnamento e di ricerca all'interno dell'università tedesca. In particolare il BFW mise in discussione l'idea, peraltro non molto definita, di democratizzazione (Demokratisierung) portata avanti dagli studenti e inoltre tentò di raffigurarsi come un'organizzazione che non faceva altro che reagire agli eccessi della controparte ${ }^{2}$.

L’idea che sostiene il lavoro di Wehrs è tentare di andare oltre la percezione superficiale, nata al momento della sua fondazione, che considerava il BFW nient'altro che un'organizzazione ultraconservatrice creata da alcuni ordinari per mantenere i loro privilegi di fronte alla "minaccia" rappresentata dalle nuove istanze giovanili. In realtà la ricerca di Wehrs dimostra con chiarezza come i professori che fondarono il BFW non facevano parte del gruppo che poteva permettersi di limitarsi a difendere qualcosa che aveva già raggiunto in passato: la maggior parte era entrata nella vita adulta intorno alla fine della seconda guerra mondiale o negli anni immediatamente successivi alla fine del conflitto e al momento della fondazione del BFW stava ancora cercando di consolidare la sua posizione all'interno dell'ambiente accademico. Questo genere di persone era quindi decisamente più determinato degli ordinari a far sentire la propria voce in un momento come gli anni Sessanta e Settanta in cui l'università tedesca

iniziava a modificare almeno in parte la propria struttura3.

$\mathrm{Al}$ momento di descrivere la nascita del BFW Wehrs sottolinea l'importanza che gli atteggiamenti eccessivamente aggressivi degli studenti hanno avuto nel far maturare la reazione dei professori e che quindi questo elemento deve essere considerato nel momento in cui si analizzano le iniziative "difensive" prese dal corpo docente". Significativa in questo senso è l'immagine scelta per la copertina del volume che rappresenta un professore berlinese il quale, dopo essere stato colpito "fisicamente" dalla contestazione studentesca, anziché ripulirsi decide di andare immediatamente in strada a denunciare l'accaduto ai passanti con un cartello al collo sul quale aveva scritto: «professore dell'università tecnica sgradito ai comunisti»5.

Pur partendo da una preoccupazione comune, secondo Wehrs i fondatori del BFW non possono essere semplicemente inseriti tutti in un'unica categoria data l'eterogeneità presente all'interno del gruppo. L'autore insiste molto sul fatto che nel BFW convivessero convinzioni politiche anche abbastanza diverse fra loro: accanto a professori vicini alla SPD, come Richard Löwenthal ${ }^{6}$, se ne trovavano altri (per esempio

${ }^{2}$ WEHRS, Nikolai, Protest der Professoren. Der «Bund Freiheit der Wissenschaft» in den 1970er Jahren, Göttingen, Wallstein Verlag, 2014, p. 14.

3 Ibidem, p. 108.

4 Ibidem, p. 71.

5 In tedesco: TU-Professor den Kommunisten missliebig.

${ }^{6}$ Richard Löwenthal (1909-1981) è stato un politologo attivo alla libera università di Berlino. È giunto all'insegnamento universitario dopo un periodo come giornalista. 
lo storico Ernst Nolte7) che si caratterizzavano per avere delle idee decisamente più conservatrici $^{8}$. In comune essi avevano l'esperienza della seconda guerra mondiale e soprattutto un fiero anticomunismo9. Un elemento chiave per le possibilità di carriera di questa generazione fu l'apertura in Germania fra il 1960 e il 1972 di venti nuove università (come quelle di Bielefeld, Bochum, Düsseldorf, Brema e Costanza) che, oltre a rispondere alla maggiore richiesta di spazi per lo studio, fornirono anche una quantità non indifferente di nuovi posti di lavoro ${ }^{10}$.

Wehrs, dopo aver ben descritto la situazione del mondo universitario tedesco in seguito alla fine della seconda guerra mondiale e il suo sviluppo fino al 1970, si occupa di descrivere minuziosamente la nascita e lo sviluppo del BFW. Particolarmente prezioso risulta essere il lavoro di ricerca su fonti primarie che ha consentito all'autore di restituire una ricca immagine delle attività del BFW in Germania. La ricerca di Wehrs copre dettagliatamente moltissimi ambiti: dalla struttura al finanziamento dell'organizzazione, dalla sua distribuzione sul territorio alle traiettorie dei suoi soci fondatori e dei membri più famosi. A volte la ricostruzione sembra insistere persino su troppi aspetti e questo è forse uno dei pochi difetti di un volume altrimenti molto ben strutturato.

Una delle parti più interessanti della ricostruzione di Wehrs riguarda la percezione del BFW nei mezzi di comunicazione tedeschi. Per quanto i membri dell'organizzazione tentassero in tutti i modi di non essere accomunati direttamente all'area conservatrice essi non riuscirono a evitare che quell'etichetta fosse loro applicata ${ }^{11}$. Questa tendenza fu propiziata anche dalla forte polarizzazione dell'opinione pubblica tedesca durante gli anni Settanta che tendeva a inserire tutto in categorie predefinite dimostrandosi poco incline a valorizzare dei punti di vista non del tutto conformi al modello tendenzialmente dicotomico vigente ${ }^{12}$.

Wehrs dedica anche molta attenzione ai tentativi del BFW di legittimare la propria esistenza insistendo molto sul rischio di una possibile "occupazione" delle università tedesche da parte delle organizzazioni comuniste sostenute dalla $\mathrm{DDR}^{13}$. In questo

\footnotetext{
7 Ernst Nolte (1923-vivente) è noto particolarmente per la sua tesi dello sviluppo dei crimini nazisti come reazione a quelli sovietici. Queste sue posizioni sono state anche cruciali per l'inizio di un dibattito (il cosiddetto Historikerstreit) che nella seconda metà degli anni Ottanta portò gli storici tedeschi a discutere apertamente sulle cause dei crimini del nazismo.

${ }^{8}$ WEHRS, Nikolai, op. cit., pp. 71-72.

9 Ibidem, p. 85 e p. 106.

${ }^{10}$ Ibidem, p. 42.

${ }^{11}$ Ibidem, p. 194.

${ }^{12} \mathrm{Su}$ questo punto si può consultare utilmente TOLOMELLI, Marica, Terrorismo e società. Il pubblico dibattito in Italia e in Germania negli anni Settanta, Bologna, Il Mulino, 2006.

${ }_{13}$ WEHRS, Nikolai, op. cit., p.272. Questo tipo di paura era ben radicato nella Germania occidentale e ciò può diventare evidente se si analizza la storia del Partito comunista tedesco
} 
senso fu spesso utilizzata un'espressione coniata da Rudi Dutschke che proponeva per il movimento una «lunga marcia attraverso le istituzioni» per descrivere la lunghezza e la complessità del processo rivoluzionario ${ }^{14}$. Per il BFW la lunga marcia non significava altro che il tentativo da parte degli "estremisti" di conquistare gradualmente posti di potere all'interno delle università, sia a livello di rappresentanza studentesca sia a livello di "infiltrazione" del corpo docente. La strategia retorica del BFW beneficiava, paradossalmente, proprio del venir meno del Sozialistischer Deutsche Studentenbund (SDS) ${ }^{15}$, l'organizzazione di cui Rudi Dutschke era stato uno dei principali rappresentanti fra il 1967 e il 1968, come guida del movimento studentesco. La fine della preminenza del SDS aveva infatti lasciato spazio a tanti piccoli gruppi di diverso orientamento che però potevano essere genericamente definiti comunisti (K-Gruppen) e che in alcune università erano effettivamente riusciti a raccogliere un certo seguito presso gli studenti.

Questa strategia fu mantenuta anche verso la fine degli anni Settanta quando il BFW si fece sostenitore dell'idea della contiguità chiara e necessaria fra parte "estremista" del movimento studentesco e organizzazioni terroristiche ${ }^{16}$. Questa per il BFW fu anche una buona occasione per portare avanti la campagna contro tutti quei professori che risultavano essere troppo accondiscendenti nei confronti degli studenti e che spesso nell'ambito della loro attività venivano definiti arbitrariamente "simpatizzanti" ${ }^{17}$ dei terroristi ${ }^{18}$. L'opposizione all'eccessiva "accondiscendenza" di

(KPD fino al 1956, dal 1968 DKP). Esso nel 1956 fu definito illegale e solo nel 1968 un partito comunista tornò a esistere nella Germania Ovest. Negli anni successivi però gli appartenenti al partito ebbero molte difficoltà nei rapporti con lo Stato, in particolare al momento di candidarsi per un impiego pubblico. Su questo argomento si può utilmente consultare RIGOLL, Dominik, Staatsschutz in Westdeutschland. Von der Entnazifizierung zur Extremistenabwehr, Göttingen, Wallstein Verlag, 2013.

${ }_{14}$ Cfr. DUTSCHKE, Rudi, Mein langer Marsch. Reden, Sachriften und Tagebücher aus zwanzig Jahren, Reinbek, Rowohlt, 1980, pp. 11-29.

${ }^{15}$ Nato come organizzazione giovanile della SPD, l'SDS si allontanò dal partito socialdemocratico in seguito alla progressiva svolta centrista di quest'ultimo. Ciò permise al SDS di porsi come forza unificante del movimento studentesco che culminò nel 1968 con il duro confronto riguardo le cosiddette leggi di emergenza (Notstandgesetze).

${ }^{16}$ WEHRS, Nikolai, op. cit., p. 404. Questo aspetto, che il BFW usava a fini propagandistici, è ancora discusso dalla storiografia. Per avere una prima idea delle diverse posizioni si può consultare KRAUSHAAR, Wolfgang, REEMTSMA, Jan Philipp, WIELAND, Karin, Rudi Dutschke Andreas Baader und die RAF, Hamburg, Hamburger Edition, 2005 e GILCHERHOLTEY, Ingrid, 1968. Eine Zeitreise, Frankfurt am Main, Suhrkamp, 2008. Per una panoramica sulla storiografia riguardante la RAF si rimanda a WEINHAUER, Klaus, «Terrorismus in der Bundesrepublik der Siebzigerjahre. Aspekte einer Sozial und Kulturgeschichte der Inneren Sicherheit», in Archiv für Sozialgeschichte, 44, 2004, pp. 219242.

17 Il termine fu usato, soprattutto dopo il 1972, per descrivere tutta quell'area che, pur non partecipando direttamente alle attività dei gruppi armati, veniva accusata di preparare loro la strada con attività intellettuali.

18 WEHRS, Nikolai, op. cit., p. 481. 
alcuni professori nei confronti delle istanze studentesche era stata infatti una delle ragioni che avevano portato alla nascita del BFW.

Nel 1973 una sentenza del Bundesverfassungsgericht (l'equivalente di fatto della Corte Costituzionale) in merito alla questione della rappresentanza ${ }^{19}$ all'interno delle università (Mitbestimmung Frage) accolse in buona parte le obiezioni sollevate dal BFW che così riuscì a raggiungere almeno una parte dei suoi obiettivi ${ }^{20}$. La storia del BFW, tuttavia, non terminò nel 1973, perché molti all'interno dell'organizzazione considerarono il "pericolo" delle istanze studentesche non ancora sparito. La spinta riformista si stava però progressivamente assopendo e infatti secondo Wehrs la riforma universitaria del 1976 conteneva molto poco delle richieste iniziali ${ }^{21}$. In questo periodo molti dei fondatori iniziarono a farsi da parte lasciando gli incarichi di vertice all'interno dell'organizzazione ${ }^{22}$. L'ultima parte della ricerca di Wehrs mostra come nella seconda metà degli anni Settanta il BFW abbandoni progressivamente la sua supposta equidistanza dai partiti politici per avvicinarsi sempre più alla conservatrice CDU partecipando direttamente alla cosiddetta Tendenzwende cioè a un profondo cambiamento nel clima politico e sociale che avrebbe chiuso la stagione delle riforme ${ }^{23}$.

Nel complesso il libro di Wehrs rappresenta sicuramente una novità per quanto riguarda la ricerca sugli anni Settanta in Germania. Esso risulta inoltre basato su un ottimo lavoro di ricerca e di documentazione. In particolare è degno di nota l'abbinamento, riuscito, di fonti scritte e di fonti orali. Come già segnalato in precedenza uno dei pochi limiti del testo è, in alcuni punti, la grande mole di informazioni fornita che può occasionalmente rendere difficoltosa la lettura.

\footnotetext{
19 Questo era uno dei punti cruciali per il movimento studentesco. Una delle proposte più accattivanti e seguite fu quella di Detlev Albers (1943-2008), studente di scienze politiche ad Amburgo e poi membro della SPD, che sosteneva la necessità di dividere in tre parti uguali la rappresentanza (studenti, professori e il cosiddetto Mittelbau, cioè grosso modo i docenti che non sono ancora diventati professori anche se la sua composizione è variabile a seconda dell'università) in modo che le decisioni fossero prese sempre cercando l'appoggio di almeno uno degli altri gruppi. Cfr. WEHRS, Nikolai, op. cit., p. 55.

${ }^{20}$ Ibidem, pp. 362-363.

${ }^{21}$ Ibidem, p. 369.

22 Ibidem, p. 389.

${ }^{23}$ Ibidem, p. 417.
} 


\section{* L'autore}

Alessandro Stoppoloni (Roma, 1989) ha completato nel luglio del 2015 il percorso per ottenere il doppio titolo nell'ambito del corso integrato italo-tedesco organizzato dall'Università di Bologna e da quella di Bielefeld discutendo una tesi dal titolo Fra teoria e pratica: la psicologia politica di Peter Brückner (1966-1978). In precedenza ha studiato presso le Università Roma Tre e Paris $\mathrm{X}$ (come studente Erasmus). In questo momento si sta occupando di temi legati alla storia della psichiatria.

URL: < http://www.studistorici.com/progett/autori/\#Stoppoloni >

\section{Per citare questo articolo:}

STOPPOLONI, Alessandro, «Recensione: Nikolai WEHRS, Protest der Professoren. Der "Bund Freiheit der Wissenschaft" in den 1970er Jahren, Göttingen, Wallstein Verlag, 2014, 539 pp.», Diacronie. Studi di Storia Contemporanea : "Se creare è definire", 29/03/2016,

URL:< http://www.studistorici.com/2016/03/29/stoppoloni_numero_25/ >

Diacronie Studi di Storia Contemporanea $\beta$ www.diacronie.it

Risorsa digitale indipendente a carattere storiografico. Uscita trimestrale. redazione.diacronie@hotmail.it

Comitato di redazione: Jacopo Bassi - Luca Bufarale - Elisa Grandi - Antonio César Moreno Cantano - Deborah Paci - Fausto Pietrancosta - Alessandro Salvador - Matteo Tomasoni - Luca Zuccolo

Diritti: gli articoli di Diacronie. Studi di Storia Contemporanea sono pubblicati sotto licenza Creative Commons 3.0. Possono essere riprodotti e modificati a patto di indicare eventuali modifiche dei contenuti, di riconoscere la paternità dell'opera e di condividerla allo stesso modo. La citazione di estratti è comunque sempre autorizzata, nei limiti previsti dalla legge. 